\title{
DISCUSSÃO SOBRE O CONCEITO DE CURTO-CIRCUITO APLICADO À ANÁLISE DO ESCOAMENTO DE DISTRIBUIDORES PARA LINGOTAMENTO CONTÍNUO*
}

\author{
Bernardo Martins Braga ${ }^{\dagger}$ \\ Carlos Eduardo Passagli Barral ${ }^{2}$ \\ Lucas Teixeira Costa ${ }^{3}$ \\ Roberto Parreiras Tavares ${ }^{4}$
}

\section{Resumo}

O curto-circuito é um dos componentes dos modelos de compartimentos utilizados na análise das curvas de Distribuição de Tempos de Residência (DTR) de distribuidores para lingotamento contínuo. Foram identificados na literatura, no entanto, diversos exemplos de seu emprego incorreto. O presente trabalho discute a interpretação física do curto-circuito, as formas de sua quantificação e as consequências nocivas de sua presença sobre a limpidez do aço e o controle da temperatura do aço no molde. Mostra-se que o método de cálculo dos parâmetros dos modelos de compartimentos deve ser alterado caso haja curto-circuito no distribuidor. É constatado que o curto-circuito deve ser evitado durante o projeto de distribuidores destinados à fabricação de aços limpos devido ao risco de perdas metálicas por macroinclusões.

Palavras-chave: Distribuidor; Curva DTR; Curto-circuito; Aço limpo.

\section{DISCUSSION ABOUT THE CONCEPT OF SHORT CIRCUIT APPLIED TO FLOW ANALYSIS OF CONTINUOUS CASTING TUNDISHES}

\section{Abstract}

Short circuit is one of components of compartment models used in the analysis of Residence Time Distribution (RTD) curves of continuous casting tundishes. It has been identified in the literature, however, several examples of misunderstandings regarding its use. This work discusses the physical interpretation of short circuit, the ways of its quantification and the harmful consequences of its presence on steel cleanness and steel temperature control in the mould. It is shown that the method of calculating parameters of compartment models must be changed if there is a short circuit in the tundish. It is pointed out that the short circuit should be avoided during the tundish design for clean steels producing due to the risk of metal losses by macroinclusions.

Keywords: Tundish; RTD curve; Short circuit; Clean steel.

1 Engenheiro Metalurgista, Mestre, Doutorando, PPGEM, UFMG, Belo Horizonte, Minas Gerais, Brasil.

2 Engenheiro Metalurgista, Mestre, Doutorando, PPGEM, UFMG, Belo Horizonte, Minas Gerais, Brasil.

3 Químico, Mestre, Doutorando, PPGEM, UFMG, Belo Horizonte, Minas Gerais, Brasil.

4 Engenheiro Metalurgista, PhD, Professor Associado, Departamento de Metalurgia e Materiais, UFMG, Belo Horizonte, Minas Gerais, Brasil. 


\section{INTRODUÇÃO}

As curvas de Distribuição de Tempos de Residência (DTR) são amplamente utilizadas em estudos empregando modelos físicos e matemáticos para analisar as características do escoamento de distribuidores para lingotamento contínuo [1-4]. Tais curvas representam a distribuição dos tempos gastos por diferentes porções de aço para percorrer o distribuidor e são obtidas através de ensaios com traçadores [5].

Um modo conveniente de extrair informações da curva DTR é sua análise por meio de modelos de compartimento. Esses modelos consideram que o distribuidor se comporta como uma combinação de componentes idealizados: região com escoamento pistonado, região com mistura perfeita, zona morta e curto-circuito [6]. As frações relativas desses componentes permitem diagnosticar problemas no perfil fluidodinâmico do distribuidor, bem como comparar o desempenho de diferentes configurações de distribuidor em termos de um objetivo específico. Frequentemente, deseja-se favorecer a flotação de inclusões não-metálicas [7].

Há na literatura especializada diversos trabalhos sobre o tema. No entanto, muitos deles não abordam o curto-circuito ou o fazem de forma incorreta tratando o curtocircuito como uma porção de volume do distribuidor. Essa má definição torna obscura a análise das consequências do curto-circuito e interfere adversamente no cálculo dos demais parâmetros dos modelos de compartimentos. Tais problemas serão superados no presente trabalho por meio da interpretação do curto-circuito em termos de uma vazão de aço que atravessa o distribuidor rapidamente.

\section{MODELOS DE COMPARTIMENTOS}

\subsection{Componentes dos Modelos de Compartimentos}

Conforme a teoria dos modelos de compartimento descrita por Levenspiel [8-9], a vazão de aço $\boldsymbol{Q}$ que alimenta o distribuidor se divide em duas parcelas: vazão de curto-circuito $\boldsymbol{Q}_{\boldsymbol{c}}$ e vazão ativa $\boldsymbol{Q}_{\boldsymbol{a}}\left(\boldsymbol{Q}=\boldsymbol{Q}_{\boldsymbol{c}}+\boldsymbol{Q}_{\boldsymbol{a}}\right)$. O volume de aço no distribuidor $\boldsymbol{V}$ também é dividido em duas parcelas: volume morto $\boldsymbol{V}_{\boldsymbol{d}}$ e volume ativo $\boldsymbol{V}_{\boldsymbol{a}}\left(\boldsymbol{V}=\boldsymbol{V}_{\boldsymbol{d}}+\right.$ $\left.V_{a}\right)$.

Fisicamente, o curto-circuito corresponde a um caminho preferencial em que o aço percorre o distribuidor com tempo de residência extremamente baixo, que é tomado como nulo durante a modelagem da curva DTR. A vazão de curto-circuito $\boldsymbol{Q}_{\boldsymbol{c}}$ quantifica a quantidade de aço que percorre tal caminho preferencial.

Vários autores [10-12] quantificam erroneamente o curto-circuito como uma fração do volume do distribuidor. Embora, de fato, se possa estimar o volume do distribuidor ocupado pelo caminho preferencial associado ao curto-circuito (tratando- como uma região de escoamento pistonado em paralelo à região ativa do distribuidor), o valor obtido possui pouco interesse prático. Independente do volume do caminho preferencial, se o tempo gasto pelo aço percorrê-lo for suficientemente curto, o aço que o atravessa não sofrerá tratamentos metalúrgicos significativos. A informação relevante ao aciarista é a quantidade de aço que atravessa o distribuidor sem ser efetivamente tratado, isto é, $\boldsymbol{Q}_{\boldsymbol{c}}$.

A região ativa corresponde à porção $\boldsymbol{V}_{\boldsymbol{a}}$ do volume interno do distribuidor disponível para que o aço escoe. $O$ aço atravessa essa região com tempos de residência finitos a uma taxa $\boldsymbol{Q}_{\boldsymbol{a}}$ sofrendo tratamentos metalúrgicos significativos. A região ativa de distribuidores é normalmente modelada pela combinação em série de uma região 
com escoamento pistonado e uma região com mistura perfeita, de volumes $\boldsymbol{V}_{\boldsymbol{p}}$ e $\boldsymbol{V}_{\boldsymbol{m}}$ respectivamente $\left(\boldsymbol{V}_{\boldsymbol{a}}=\boldsymbol{V}_{\boldsymbol{p}}+\boldsymbol{V}_{\boldsymbol{m}}\right)$.

Por fim, as zonas mortas representam regiões internas do distribuidor inacessíveis ao escoamento do aço. Essas zonas possuem volume $\boldsymbol{V}_{\boldsymbol{d}}$. Outros autores, como Fogler [13] e Sahai e Emi [4,14], consideram que as zonas mortas trocam matéria lentamente com a região ativa do distribuidor. Foge do escopo deste trabalho uma discussão detalhada sobre as zonas mortas.

\subsection{Exemplos de Modelos de Compartimentos}

A Figura 1a apresenta um modelo de compartimentos composto por uma região de escoamento pistonado em série com uma região de mistura perfeita. A Figura 1b apresenta a extensão do modelo anterior com a introdução de um curto-circuito. As curvas DTR correspondentes aos dois modelos podem ser obtidas por meio de um balanço de massa dinâmico [5,13]. A solução analítica foi obtida por Levenspiel [8-9] e é apresentada graficamente na Figura 2.

a)

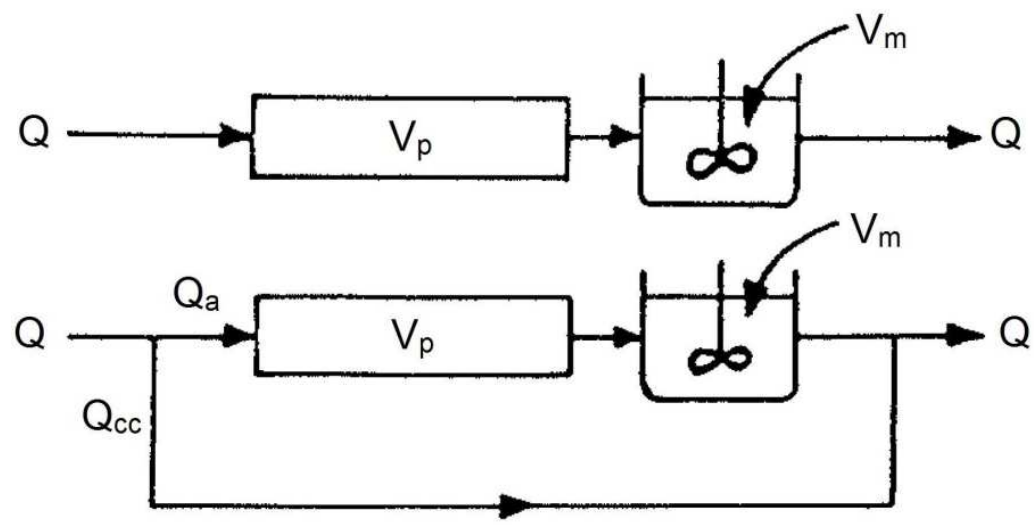

Figura 1. Modelos de compartimentos: a) região de escoamento pistonado em série com uma região de mistura perfeita. perfeita; b) extensão do modelo anterior com a introdução de um curto-circuito. Fonte: Adaptado de Singh e Koria [6]. 
a)

b)

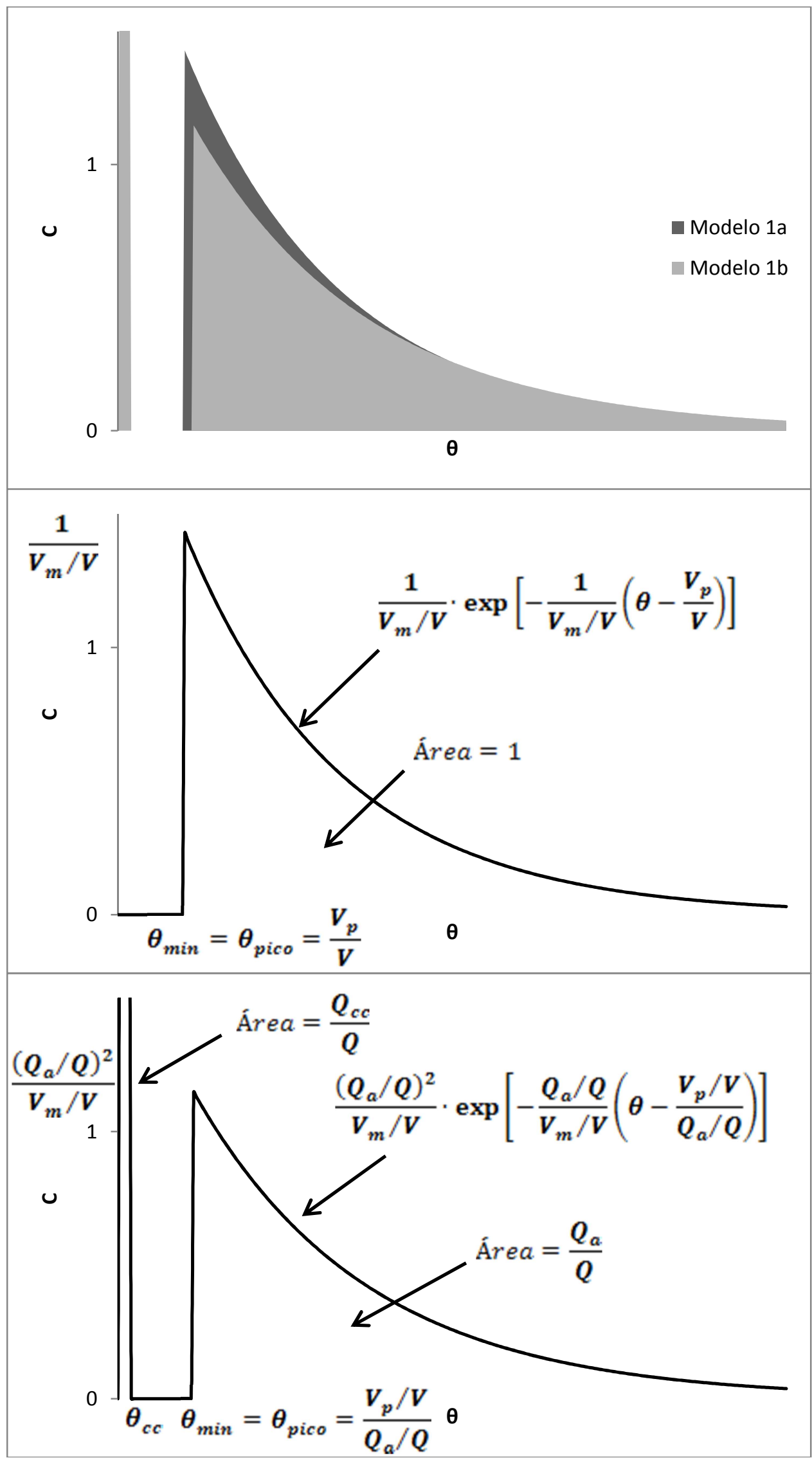

Figura 2. Representação gráfica de curvas DTR para modelos de compartimentos da Figura 1: a) curvas sobrepostas para $V_{p} / V=0,2, V_{m} / V=0,8, Q_{c c} / Q=0,1$ e $Q_{a} / Q=0,9$; b) solução analítica detalhada para modelo $1 \mathrm{a} ; \mathrm{c})$ solução analítica detalhada para modelo $1 \mathrm{~b}$. 
Nessa figura, $\boldsymbol{C}$ é a função DTR dos modelos representados, $\boldsymbol{\theta}$ o tempo adimensional $(\boldsymbol{\theta}=\boldsymbol{t} / \overline{\boldsymbol{t}}), \boldsymbol{t}$ o tempo (s), $\overline{\boldsymbol{t}}$ o tempo nominal médio de residência (s), $\overline{\boldsymbol{t}}=\boldsymbol{V} / \boldsymbol{Q}, \boldsymbol{V}$ o volume de aço no distribuidor $\left(\mathrm{m}^{3}\right), \boldsymbol{Q}$ a vazão volumétrica de aço no distribuidor $\left(\mathrm{m}^{3} / \mathrm{s}\right), \boldsymbol{V}_{\boldsymbol{p}} / \boldsymbol{V}$ a fração de volume do distribuidor com escoamento pistonado, $\boldsymbol{V}_{\boldsymbol{m}} / \boldsymbol{V}$ a fração de volume do distribuidor com mistura perfeita, $\boldsymbol{Q}_{c c} / \boldsymbol{Q}$ a fração de vazão do distribuidor que passa pelo curto-circuito e $\boldsymbol{Q}_{\boldsymbol{a}} / \boldsymbol{Q}$ a fração da vazão que atravessa a região ativa do distribuidor. A função $\boldsymbol{C}$ é definida de forma que a área sob a curva DTR entre dois pontos quaisquer, $\boldsymbol{\theta}_{\mathbf{1}}$ e $\boldsymbol{\theta}_{\mathbf{2}}$, expressa a fração de aço que demanda um tempo adimensional entre $\boldsymbol{\theta}_{\mathbf{1}}$ e $\boldsymbol{\theta}_{\mathbf{2}}$ para percorrer 0 distribuidor. Como todo aço que adentra o distribuidor eventualmente o deixa, a área sob todo curva DTR é sempre unitária, a menos de erros experimentais ou numéricos.

A análise das Figuras 1a, 2a e 2b permite identificar o seguinte método para cálculo dos parâmetros do modelo de compartimentos da Figura 1a:

$$
\begin{aligned}
& \left(\frac{V_{p}}{V}\right)_{1 a}=\theta_{\text {min }}=\theta_{\text {pico }} \\
& \left(\frac{V_{m}}{V}\right)_{1 a}=1-\left(\frac{V_{p}}{V}\right)_{1 a}
\end{aligned}
$$

Em que $\boldsymbol{\theta}_{\min }$ é o tempo adimensional mínimo para o aço atravessar a zona ativa do distribuidor e $\boldsymbol{\theta}_{\text {pico }}$ o tempo adimensional em que a parte "ativa" da DTR apresenta seu máximo.

De forma similar, o método a seguir para cálculo dos parâmetros do modelo de compartimentos da Figura 1 b é obtido pela análise das Figuras 1b, 2a e 2c:

$$
\begin{gathered}
\left(\frac{Q_{c c}}{Q}\right)_{1 b}=A_{0, \theta_{c c}} \\
\left(\frac{Q_{a}}{Q}\right)_{1 b}=1-\left(\frac{Q_{c c}}{Q}\right)_{1 b} \\
\left(\frac{V_{p}}{V}\right)_{1 b}=\left(\frac{Q_{a}}{Q}\right)_{1 b} \cdot \theta_{\text {min }}=\left(\frac{Q_{a}}{Q}\right)_{1 b} \cdot \theta_{\text {pico }} \\
\left(\frac{V_{m}}{V}\right)_{1 b}=1-\left(\frac{V_{p}}{V}\right)_{1 b}
\end{gathered}
$$

Em que $\boldsymbol{A}_{\mathbf{0}, \boldsymbol{\theta}_{c c}}$ é a área da curva DTR sobre região correspondente ao curto-circuito e $\boldsymbol{\theta}_{c c}$ o tempo adimensional da curva DTR em que o curto-circuito termina. Para o cálculo de $\boldsymbol{\theta}_{\text {min }}$ e $\boldsymbol{\theta}_{\text {pico }}$, analisa-se a DTR a partir de $\boldsymbol{\theta}_{\boldsymbol{c} \boldsymbol{c}}$.

Nota-se que a equação de cálculo da fração de volume da região com escoamento pistonado $\boldsymbol{V}_{\boldsymbol{p}} / \boldsymbol{V}$ necessita ser modificada na presença de curto-circuito. Mais especificadamente, o curto-circuito reduz a vazão que atravessa a região com escoamento pistonado fazendo aumentar o tempo adimensional mínimo $\boldsymbol{\theta}_{\min }$ da curva DTR após $\boldsymbol{\theta}_{c c}$ (Figura $2 \mathrm{a}$ ).

Como curvas DTR reais normalmente apresentam $\boldsymbol{\theta}_{\min } \neq \boldsymbol{\theta}_{\text {pico }}$, alguns autores consideram que a região com escoamento pistonado do modelo de compartimento apresenta dispersão axial [3,11-12]. Sahai e Ahuja [15], citados por Mazumdar e Guthrie [3], propuseram a seguinte fórmula para o cálculo de sua fração volumétrica na ausência de curto-circuito: 


$$
\left(\frac{V_{p}}{V}\right)_{S A}=\frac{\theta_{\min }+\theta_{\text {pico }}}{2}
$$

A equação anterior pode ser generalizada de forma direta para distribuidores com curto-circuito - compare as equações (1), (5) e (7):

$$
\left(\frac{V_{p}}{V}\right)_{S A G}=\left(\frac{Q_{a}}{Q}\right)_{\text {modelo }} \cdot \frac{\theta_{\text {min }}+\theta_{\text {pico }}}{2}
$$

A fração de vazão ativa $\boldsymbol{Q}_{\boldsymbol{a}} / \boldsymbol{Q}$ presente na equação anterior deve ser avaliada em conformidade com o modelo de compartimentos usado. Por exemplo, para os modelos de Szekely e llegbusi [1], Singh e Koria [6] e Sahai e Ahuja [15] deve-se aplicar a equação (4): $\boldsymbol{Q}_{\boldsymbol{a}} / \boldsymbol{Q}=\mathbf{1}-\boldsymbol{Q}_{\boldsymbol{c c}} / \boldsymbol{Q}$. Já para o modelo de Sahai e Emi [4,14]: $\boldsymbol{Q}_{\boldsymbol{a}} / \boldsymbol{Q}=\boldsymbol{A}_{\boldsymbol{\theta}_{\min }, 2}$, sendo $\boldsymbol{A}_{\boldsymbol{\theta}_{\min }, \mathbf{2}}$ a área sob a curva DTR entre $\boldsymbol{\theta}_{\min }$ e $\boldsymbol{\theta}=\mathbf{2}$.

\section{CONSEQUÊNCIAS NOCIVAS DO CURTO-CIRCUITO}

A seguir será descrito o impacto do curto-circuito sobre a limpidez do aço. Rigorosamente, as considerações feitas só são válidas para uma máquina de lingotamento operando em regime estacionário, pois essa é a condição de obtenção das curvas DTR.

Durante trocas de panelas, o perfil de escoamento do aço no distribuidor pode ser afetado significativamente por razões diversas: alteração do nível de metal no distribuidor, mudança da velocidade de lingotamento e variações expressivas da temperatura do aço que alimenta o distribuidor [4]. Portanto, esse momento do lingotamento deve ser analisado em separado por outras técnicas [16-17].

\subsection{Limpidez do Aço}

Qualitativamente, a presença do curto-circuito tem dois efeitos antagônicos sobre a flotação de inclusões não-metálicas. Por um lado, o curto-circuito faz com que a fração de aço $\boldsymbol{Q}_{\boldsymbol{c}} / \boldsymbol{Q}$ deixe o equipamento sem que sofra praticamente nenhum tratamento metalúrgico, isto é, sem que haja flotação significativa de inclusões. Por outro, o tempo médio de residência da região ativa do distribuidor $\left(\overline{\boldsymbol{t}}_{\boldsymbol{a}}=\boldsymbol{V}_{\boldsymbol{a}} / \boldsymbol{Q}_{\boldsymbol{a}} \geq\right.$ $\left.\boldsymbol{V}_{\boldsymbol{a}} / \boldsymbol{Q}\right)$ é aumentado favorecendo a flotação de inclusões presentes nessa porção de aço.

O resultado líquido desses dois efeitos é função da DTR do distribuidor e do tamanho da inclusão não-metálica. No entanto, é aparente que um curto-circuito relativamente pequeno (por exemplo, $\boldsymbol{Q}_{c c} / \boldsymbol{Q}=\mathbf{0 , 0 5}$ ) não afeta significativamente a eficiência média de flotação de inclusões do distribuidor. Portanto, é possível que alguma configuração de distribuidor com curto-circuito apresente maior eficiência média de flotação de inclusões que outras configurações do mesmo distribuidor sem curto-circuito.

Apesar do exposto, o curto-circuito deve, em geral, ser evitado durante o projeto de distribuidores destinados à fabricação de aços limpos. A razão é simples. O metal líquido pode apresentar macroinclusões de fontes diversas (tratamento de cálcio incorreto, fragmentos de refratários, reações de reoxidação e emulsificação da escória) que, se presentes no produto solidificado, podem levar à rejeição da corrida. Essas macroinclusões, pelo seu grande tamanho, flotariam facilmente no distribuidor 
(lei de Stokes). Entretanto a presença de um curto-circuito leva inevitavelmente uma fração dessas inclusões ao veio aumentando o risco de perdas metálicas.

A fim de sedimentar o que foi exposto, é apresentado a seguir um exemplo numérico. A porcentagem de inclusões passantes para o molde $\% \boldsymbol{P}$ pode ser expressa por:

$$
\% P=100 \cdot\left(1-\frac{Q_{a}}{Q} \cdot \frac{\% F_{a}}{100}-\frac{Q_{c c}}{Q} \cdot \frac{\% F_{c c}}{100}\right)
$$

Em que $\% \boldsymbol{F}_{\boldsymbol{a}}$ é a porcentagem de inclusões flotadas pelo aço que atravessa a região ativa do distribuidor e $\% \boldsymbol{F}_{c c}$ a porcentagem de inclusões flotadas pelo aço que atravessa o curto-circuito. Por razões físicas, a equação (9) está sujeita a $\% \boldsymbol{F}_{c c} \ll$ $\% \boldsymbol{F}_{\boldsymbol{a}}$ e $\boldsymbol{Q}_{\boldsymbol{c}} / \boldsymbol{Q}+\boldsymbol{Q}_{\boldsymbol{a}} / \boldsymbol{Q}=\mathbf{1}$.

A Figura 3 apresenta curvas de nível para a equação (9) assumindo $\% \boldsymbol{F}_{\boldsymbol{c c}} \approx \mathbf{0}, \mathbf{0 5}$. $\% \boldsymbol{F}_{\boldsymbol{a}}$. Os pontos "A", "B", "C" e "D" da figura representam quatro comportamentos possíveis de configurações de distribuidor em relação à flotação de inclusões nãometálicas de tamanho médio ( $\approx 60-80 \mu \mathrm{m}[18])$. A configuração de distribuidor " $A$ " ilustra uma condição típica de distribuidor sem curto-circuito $\left(\boldsymbol{Q}_{c c} / \boldsymbol{Q}=\mathbf{0}\right)$ enquanto as configurações "B", "C" e "D" representam condições com curto-circuito $\left(\boldsymbol{Q}_{c c} / \boldsymbol{Q}>\right.$ 0).

A configuração "B" $(\% \boldsymbol{P}=\mathbf{3 5}, \mathbf{3})$ possui a maior porcentagem de inclusões-nãometálicas médias passantes para o molde dentre as quatros configurações indicadas na Figura 3. Essa configuração possui um perfil fluidodinâmico desfavorável à flotação de inclusões na região ativa do distribuidor $\left(\% \boldsymbol{F}_{\boldsymbol{a}}=\mathbf{7 0}\right)$ e o curto-circuito $\left(\boldsymbol{Q}_{c c} / \boldsymbol{Q}=\mathbf{0}, \mathbf{0 8}\right)$ apenas agrava o problema de limpidez do aço.

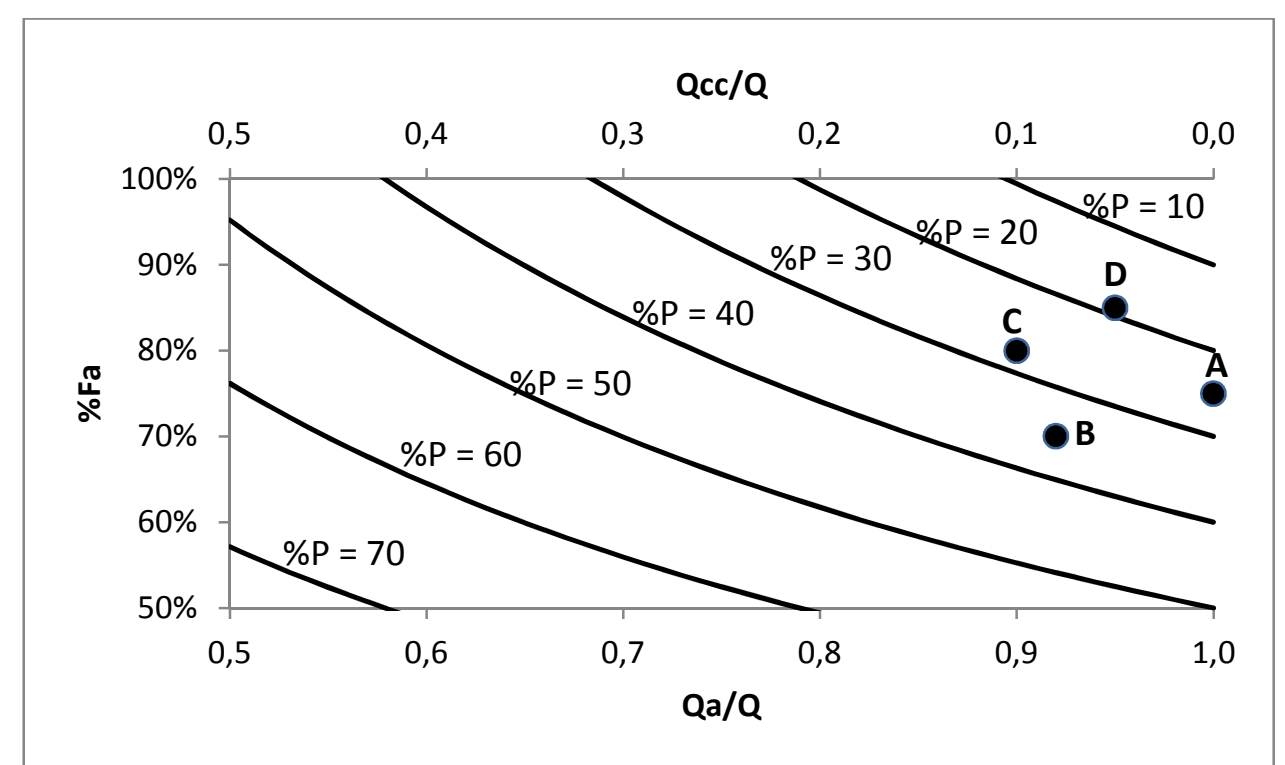

Figura 3. Curvas de nível da porcentagem de inclusões não-metálicas passantes para o molde (\%P) em função da porcentagem inclusões flotada na região ativa do distribuidor $\left(\% F_{a}\right)$ e da magnitude do curto-circuito $\left(Q_{c c} / Q\right)$, ou equivalentemente da vazão ativa $\left(Q_{a} / Q\right)$.

A configuração "C" $(\% \boldsymbol{P}=\mathbf{2 7}, \mathbf{6})$ possui maior índice de inclusões médias passantes para o molde que a configuração "A" $(\% \boldsymbol{P}=\mathbf{2 5}, \mathbf{0})$, embora sua região ativa seja capaz de flotar mais inclusões médias $\left(\% \boldsymbol{F}_{\boldsymbol{a}}=\mathbf{8 0}\right)$ que a configuração "A" $\left(\% \boldsymbol{F}_{\boldsymbol{a}}=\right.$ 75). A causa disso é o curto-circuito da configuração "C" $\left(\boldsymbol{Q}_{c c} / \boldsymbol{Q}=\mathbf{0}, \mathbf{1 0}\right)$ que arrasta inclusões para o molde. A configuração "D" $(\% \boldsymbol{P}=\mathbf{1 9}, \mathbf{0})$, por sua vez, possui menor 
índice de inclusões médias passantes para o molde entre todas as configurações, apesar dessa configuração possuir curto-circuito $\left(\boldsymbol{Q}_{c c} / \boldsymbol{Q}=\mathbf{0 , 0 5}\right)$. A razão é seu perfil fluidodinâmico favorável à flotação de inclusões $\left(\% \boldsymbol{F}_{\boldsymbol{a}}=\mathbf{8 5}\right)$ na região ativa do distribuidor. Fica claro que o curto-circuito influência a eficiência de flotação de inclusões de tamanho médio no distribuidor, mas sua presença não necessariamente indica que uma configuração é ruim em relação a esse tipo de inclusão.

Por questões de escala, a flotação de macroinclusões $\left(\% \boldsymbol{F}_{\boldsymbol{a}} \approx \mathbf{1}\right)$ não pode ser analisada adequadamente pela Figura 3. A Figura 4 apresenta uma nova curva nível para a equação $(9)$ dando ênfase à região de inclusões de tamanho grande $(\approx 100$ $120 \mu \mathrm{m})[18]$.

O comportamento das configurações de distribuidor em relação à passagem de inclusões não-metálicas grandes para o molde é diferente daquele para inclusões médias. Para macroinclusões, a quantidade de partículas passantes para o molde está intimamente relacionada à magnitude do curto-circuito. De fato, para $\% \boldsymbol{F}_{\boldsymbol{a}} \approx \mathbf{1} \mathrm{e}$ $\% \boldsymbol{F}_{c c} \approx \mathbf{0}$, a equação (9) se simplifica para:

$$
\% P \approx 100 \cdot\left(1-\frac{Q_{a}}{Q}\right)=100 \cdot \frac{Q_{c c}}{Q}
$$

o que demonstra a relevância prática do valor de $\boldsymbol{Q}_{c c} / \boldsymbol{Q}$ obtido pela DTR.

$A$ equação (10) se aplica razoavelmente bem às configurações de distribuidor "A" $\left(\boldsymbol{Q}_{c c} / \boldsymbol{Q}=\mathbf{0}, \quad \% \boldsymbol{P}=\mathbf{2}\right), \quad$ "C" $\left(\boldsymbol{Q}_{c c} / \boldsymbol{Q}=\mathbf{0}, \mathbf{1 0}, \quad \% \boldsymbol{P}=\mathbf{1 0}, \mathbf{4}\right) \quad \mathrm{e}$ "D" $\left(\boldsymbol{Q}_{c c} / \boldsymbol{Q}=\mathbf{0 , 0 5}\right.$, $\% \boldsymbol{P}=\mathbf{4 , 8}$ ) da Figura 4 , pois suas regiões ativas são capazes de flotar praticamente todas as macroinlcusões $\left(\% \boldsymbol{F}_{\boldsymbol{a}} \geq \mathbf{9 8}\right)$. Para a configuração "B" $\left(\boldsymbol{Q}_{c c} / \boldsymbol{Q}=\mathbf{0}, \mathbf{0 8}\right.$, $\% P=12,2)$, a equação (10) apresenta desvio mais alto, devido à má flotação de macroinclusões pela região ativa dessa configuração $\left(\% \boldsymbol{F}_{\boldsymbol{a}}=\mathbf{9 5}\right)$.

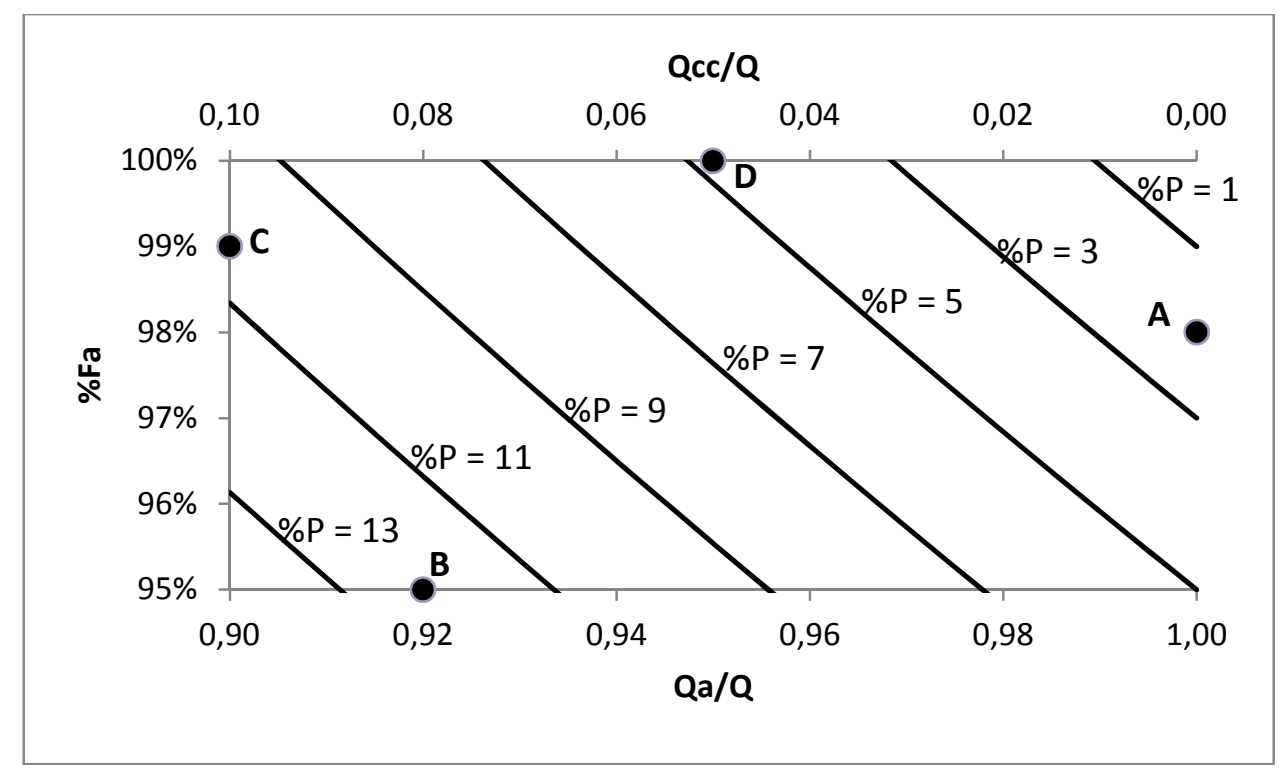

Figura 4. Curvas de nível da porcentagem de inclusões não-metálicas passantes para o molde (\%P) em função da porcentagem inclusões flotada na região ativa do distribuidor $\left(\% \mathrm{~F}_{\mathrm{a}}\right)$ e da magnitude do curto-circuito $\left(Q_{c c} / Q\right)$, ou equivalentemente da vazão ativa $\left(Q_{a} / Q\right)$.

\subsection{Controle da Temperatura do Aço no Molde}

Tang, Krobath e Lueftenegger [19] apontam que a presença de curto-circuito no distribuidor é prejudicial ao processo de lingotamento, pois pode resultar em uma 
temperatura de lingotamento irregular. Essa afirmativa necessita ser interpretada com cautela.

O curto-circuito faz com que alterações repentinas da temperatura do aço que alimenta o distribuidor sejam sentidas rapidamente no molde. Essas alterações repentinas ocorrem durante trocas de panelas e podem surgir também durante 0 regime estacionário oriunda da má homogeneidade do aço na panela. Dois pontos aqui cabem ressalva.

Em primeiro lugar, a DTR pode não ser válida em casos de alterações significativas da temperatura que alimenta o distribuidor devido à alteração do perfil de escoamento do aço no distribuidor por convecção natural [4,17].

Em segundo, não é claro se a flutuação de temperatura sentida no molde é significativa. Assumindo que o aço atravessa o curto-circuito instantaneamente e sem trocar calor com a região ativa do distribuidor e que a DTR do distribuidor apresenta $\boldsymbol{\theta}_{\min }>\boldsymbol{\theta}_{\boldsymbol{c}}$, um balanço de energia no veio leva a:

$$
\rho \cdot Q \cdot C_{p} \cdot\left(T_{s a i}-T_{r e f}\right)=\rho \cdot Q_{c c} \cdot C_{p} \cdot\left(T_{c c}-T_{r e f}\right)+\rho \cdot Q_{a} \cdot C_{p} \cdot\left(T_{a}-T_{r e f}\right)
$$

Em que $\boldsymbol{\rho}$ é a densidade do aço $\left(\mathrm{kg} / \mathrm{m}^{3}\right), \boldsymbol{C}_{\boldsymbol{p}}$ a capacidade calorífica do aço a pressão constante $\left(\mathrm{J} \mathrm{kg}^{-1} \stackrel{\circ}{ }^{-1}\right), \boldsymbol{T}_{\text {sai }}$ a temperatura do aço que deixa o distribuidor $\left({ }^{\circ} \mathrm{C}\right), \boldsymbol{T}_{\text {ref }}$ uma temperatura de referência arbitrária $\left({ }^{\circ} \mathrm{C}\right), \boldsymbol{T}_{\boldsymbol{c c}}$ a temperatura nova do aço que atravessa o curto-circuito e $\boldsymbol{T}_{\boldsymbol{a}}$ a temperatura antiga do aço no distribuidor que atravessa sua região ativa.

Tomando $\boldsymbol{T}_{\boldsymbol{r} \boldsymbol{e f}}=\boldsymbol{T}_{\boldsymbol{a}}$ e dividindo ambos os lados da equação (11) por $\boldsymbol{\rho} \cdot \boldsymbol{Q} \cdot \boldsymbol{C}_{\boldsymbol{p}}$, chega-se a seguinte expressão para o cálculo da flutuação de temperatura $\Delta \boldsymbol{T}_{\boldsymbol{m}}$ $\left(=\boldsymbol{T}_{\text {sai }}-\boldsymbol{T}_{\boldsymbol{a}}\right)$ sentida no molde $\left({ }^{\circ} \mathrm{C}\right)$ :

$$
\Delta T_{m}=\frac{Q_{c c}}{Q} \cdot \Delta T_{d}
$$

Em que $\Delta \boldsymbol{T}_{\boldsymbol{d}}\left(=\boldsymbol{T}_{\boldsymbol{c} \boldsymbol{c}}-\boldsymbol{T}_{\boldsymbol{a}}\right)$ é a magnitude da variação repentina de temperatura do aço que alimenta o distribuidor $\left({ }^{\circ} \mathrm{C}\right)$.

Por exemplo, uma alteração de $10^{\circ} \mathrm{C}$ do aço que alimenta um distribuidor com curtocircuito $\boldsymbol{Q}_{c c} / \boldsymbol{Q}=\mathbf{0 , 1 0}$ seria sentida no molde como uma flutuação rápida de aproximadamente $1^{\circ} \mathrm{C}$. A estimação feita é conservadora. Na prática, o aço que atravessa o curto-circuito troca algum calor com o restante do distribuidor e a flutuação de temperatura sentida no molde é menor que aquela calculada pela equação (12).

\section{CONCLUSÃO}

O presente trabalho discutiu a interpretação física do curto-circuito, as consequências nocivas de sua presença e formas de sua quantificação. Os seguintes pontos merecem destaque:

- Fisicamente, o curto-circuito corresponde a um caminho preferencial em que o aço percorre o distribuidor com tempo de residência extremamente baixo;

- O curto-circuito deve ser quantificado em termos da vazão de aço que atravessa o caminho preferencial associado ao curto-circuito e não em termos de uma porção de volume do distribuidor. O tratamento correto torna simples e intuitiva a análise das consequências do curto-circuito tanto em relação à limpidez do aço quanto ao controle da temperatura do aço no molde; 
- A forma de cálculo do volume da região do distribuidor com escoamento pistonado deve ser modificada na presença do curto-circuito;

- A presença do curto-circuito não necessariamente indica que uma configuração é ruim em relação à eficiência média de flotação de inclusões não-metálicas;

- O curto-circuito deve, em geral, ser evitado durante o projeto de distribuidores destinados à fabricação de aços limpos devido ao risco de perdas metálicas por macroinclusões.

\section{Agradecimentos}

O apoio financeiro da FAPEMIG - Fundação de Amparo à Pesquisa do Estado de Minas Gerais, Brasil - na forma da bolsa de pesquisa para R. Tavares, Processo № PPM-00118-13, é agradecido.

Os autores agradecem também o apoio da CAPES/PROEX ao programa de pósgraduação PPGEM/UFMG.

A bolsa de doutorado, № 1487157, concedida pela CAPES a B. Braga é agradecida.

\section{REFERÊNCIAS}

1 Szekely J, llegbusi OJ. The physical and mathematical modeling of tundish operations. New York: Springer-Verlag; 1989.

2 Costa Neto JG. Otimização do fluxo de aço no distribuidor do lingotamento contínuo [tese de doutorado]. Campinas: Universidade Estadual de Campinas; 1997.

3 Mazumdar D, Guthrie RIL. The physical and mathematical modelling of continuous casting systems. ISIJ International. 1999;39(6):524-547.

4 Sahai Y, Emi T. Tundish technology for clean steel production. Singapore: World Scientific; 2007.

5 Danckwerts PV. Continuous flow systems. Distribution of residence times. Chemical Engineering Science. 1995;50(24):3857-3866.

6 Singh S, Koria SC. Model study of the dynamics ot flow of steel melt in the tundish. ISIJ International. 1993;33(12):1228-1237.

7 Alves JG. Melhoria no padrão de escoamento do aço líquido no distribuidor do lingotamento contínuo 01 da APERAM Inox América do Sul [dissertação de mestrado]. Belo Horizonte: Universidade Federal de Minas Gerais; 2014.

8 Levenspiel O. Chemical reaction engineering. 3 ed. New York: John Wiley \& Sons; 1998.

9 Levenspiel O. Tracer technology: modeling the flow of fluids. New York: Springer; 2012.

10 Furtado HS, Fialho MM, Silva CA, Nascimento ZD, Borges RN. Development of a new tundish design. In: Instituto Argentino de Siderurgia. Anais do $16^{\text {th }}$ Steelmaking Conference; 2007; Rosario, Argentina. Buenos Aires: IAS; 2007. p. 393-402

11 Kumar A, Koria SC, Mazumdar D. Basis for systematic hydrodynamic analysis of a multi-strand tundish. ISIJ International. 2007;47(11):1618-1624.

12 Machado FD. Modelagem física de remoção de inclusões em distribuidor de lingotamento contínuo de tarugos [dissertação de mestrado]. Porto Alegre: Universidade Federal do Rio Grande do Sul; 2014.

13 Fogler HS. Elements of chemical reaction engineering. 4 ed. Upper Saddle River: Prentice Hall; 2005.

14 Sahai Y, Emi T. Melt flow characterization in continuous casting tundishes. ISIJ International. 1996;36(6):667-672.

15 Sahai Y, Ahuja R. Fluid-flow and mixing of melt in steelmaking tundishes. Ironmaking \& Steelmaking. 1986;13(5):241-247. 
16 HUANG X, THOMAS BG. Intermixing model of continuous casting during a grade transition. Metallurgical and Materials Transactions B. 1996;27(4):617-632.

17 Chattopadhyay K, Isac M, Guthrie RIL. Modelling of Non-isothermal Melt Flows in a Four Strand Delta Shaped Billet Caster Tundish Validated by Water Model Experiments. ISIJ International. 2012;52(11):2026-2035.

18 Chattopadhyay K, Isac M, Guthrie RIL. Effect of flow modifiers on liquid metal cleanliness in four-strand delta shaped billet caster tundish. Ironmaking and Steelmaking. 2012;39(6):454-462.

19 Tang Y, Krobath M, Lueftenegger A. Tundish design using computational fluid dynamics. In: Anais do $49^{\text {th }}$ International Colloquium on Refractories; 2006; Aachen, Germany. Düsseldorf: Verlag Stahleisen; 2006. p. 189-193. 